\title{
Clinical review of intravenous opioids in acute care
}

\author{
Mark J. McKeen ${ }^{1,2}$ and Sadeq A. Quraishi ${ }^{1,2^{*}}$ \\ *Correspondence: squraishi@partners.org \\ 'Massachusetts General Hospital, Boston, MA, USA. \\ ${ }^{2}$ Harvard Medical School, Boston, MA, USA.
}

\begin{abstract}
Opioids are routinely used in the management of moderate to severe pain. However, in the acute care setting, consistently undertreated and suboptimally managed pain continues to be a problem. Given the potential for opioids to result in lifethreatening situations, and the increasing complexity of the patients that are cared for in acute care facilities, a thorough understanding of intravenous opioid therapy is a necessity for hospital-based clinicians. Opioids can be classified by chemical structure and/or drug effect (i.e. action on $\mathrm{Mu}$, Kappa, and Delta opioid receptors throughout the body). Endogenous opioids include endorphins, enkephalins, and dynorphins, which moderate the body's natural response to pain. Commonly used exogenous intravenous opioids include morphine, hydromorphone, fentanyl, meperidine, methadone, buprenorphine, butorphanol, and nalbuphine, which vary greatly in potency, duration of action, metabolism, and in their adverse effect profile. A growing body of evidence suggests that patient controlled analgesia may be superior to conventional methods (need for analgesia determined by clinicians) of treating pain in the acute care setting. Newer pump delivery systems may also decrease the risks of human and equipment errors as well as enhance patient safety and satisfaction. The purpose of this review is to help guide clinicians in the safe and effective management of pain in patients requiring intravenous opioid therapy in the acute care setting.
\end{abstract}

Keywords: Acute pain, acute care, opioid, PCA

\section{Introduction}

Intravenous opioid therapy for the treatment of pain is incredibly common in the acute care setting. Opioids are essential to the management of moderate to severe pain in both, medical and surgical patients (Figure 1). However, given the increasing number of options available to clinicians, along with concerns about drug interactions and adverse effects of these complex drugs [1], the task of treating pain with intravenous opioids in the acute care setting can be challenging. Moreover, issues of patient dissatisfaction with pain management and iatrogenic overdose are major problems in hospitals today $[2,3]$.

The concept of pain as a $5^{\text {th }}$ vital sign continues to gain broader acceptance [4], and often serves as an indicator of healthcare quality. In fact, the Centers for Medicare and Medicaid use HCAHPS (Hospital Consumer Assessment of Healthcare Providers and Systems), a standardized survey tool to evaluate adult patient perceptions of quality of care in acute care facilities [5]. The ultimate goals of this program are to provide financial incentives to hospitals to provide higher quality of care and also to provide patients with the opportunity to compare the quality of care provided at competing acute care hospitals. In this survey, 2 of the 18 core experience questions directly relate to pain management and 4 of the 18 are indirectly related to it [5]. As such, it is clear that providing appropriate pain control is a major focus of current healthcare reform efforts.

In recent years, morbidity and mortality related to opioid overdose has become an increasingly common problem. From 1999 to 2006, the number of poisoning deaths in the United States has nearly doubled, largely because of overdose deaths involving prescription opioid painkillers [6]. Furthermore, the use of prescription opioids has quadrupled from 2004 to 2007, and opioid overdose is now the leading cause of unintentional death in at least 5 States [6]. The persistent increases in prescription opioid overdoses pose a unique and significant challenge for healthcare providers, who often must balance the need to effectively manage pain in challenging patients and avoid accidental overdoses [2].

Pain is an incredibly common complaint on inpatient medical wards. This is perhaps not surprising, given that pain intensity tends to be underestimated by care providers [3]. In fact, a 2004 survey amongst patients in the acute care setting demonstrated that up to $43 \%$ of patients experienced inadequately treated pain, while $12 \%$ complained of unbearable pain during hospitalization [3]. Furthermore, over $20 \%$ of elderly care and general medical patients reported pain scores $\geq 6$ on visual analog scale (VAS) assessment despite provider-guided pain management [3]. In the surgical setting, up to $86 \%$ of postoperative patients reported significant periods of undertreated pain of moderate severity [7]. Consequently, routine self-reports by patients may be a better measure of pain than assessments by a nurse or physician [8].

While opioids are frequently prescribed to hospitalized 


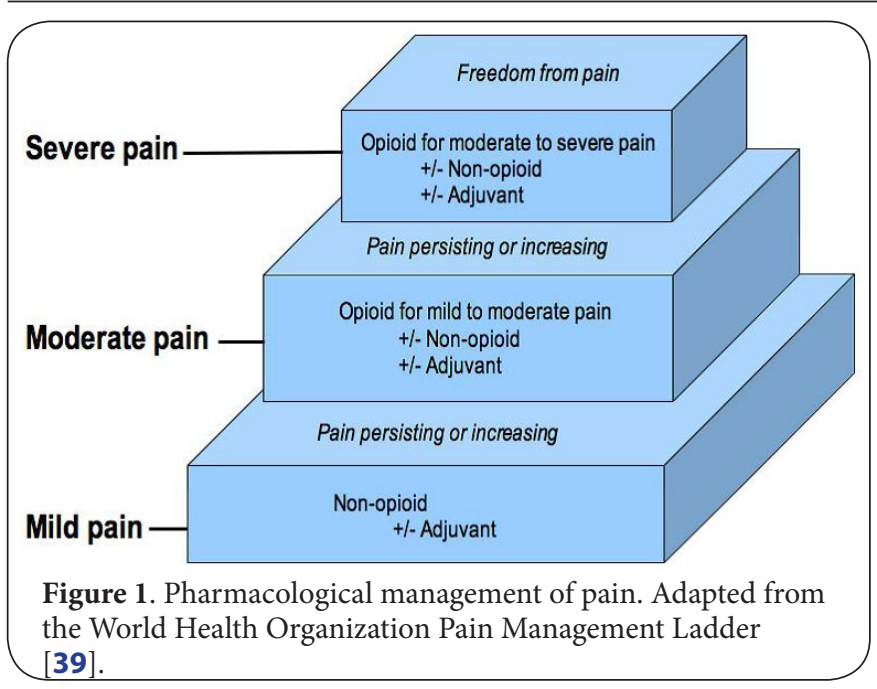

patients for moderate to severe pain, Murnion et al., studied the specifics of inpatient opioid utilization at a tertiary care hospital [9]. Of 463 adult patients admitted to the general medicine, orthopedic, and oncology wards, $45 \%$ were prescribed opioids during their hospitalization. Overall, oxycodone was the most frequently prescribed opioid (51\%). The most commonly administered intravenous opioid was morphine, representing $29 \%$ of all prescribed opioids. Approximately $65 \%$ of patients who received opioids, reported incomplete pain relief during the hospitalization, yet, the majority of these patients were not administered all of their "allowable" opioid doses by healthcare providers. In addition, identified barriers to optimal pain management strategies by providers in the acute care setting included: 1 ) limited knowledge of pain assessment techniques 2) limited knowledge of opioid dose titration; and 3) misdirected attitudes towards opioids, pain relief, and risk of chemical dependence [10]. In addition to these factors, it is also important to consider the concept of "opioid pseudoaddiction" from inadequate treatment of chronic pain, racism, sexism, and opiophobia on the part of inexperienced clinicians as real barriers to effective acute pain management in the inpatient setting [45].

Given the high likelihood of undertreating acute pain in the hospital setting, despite the increasing frequency of intravenous opioid use as treatment, it is essential for clinicians to have a sound understanding of these drugs to maximize their appropriate use. With these considerations in mind, our goal is to provide a concise review of the classification, basic pharmacology, and dosing strategies related to the most commonly available intravenous opioids in clinical use.

\section{Historical perspective}

The first historical reference to opium dates back to the $3^{\text {rd }}$ century B.C. where Arab physicians were experienced in its uses [11]. Opium use became more widespread in the Middle Ages, and in 1806 Freidrich Wilhelm Adam Serturner reported the isolation of a pure substance in opium that he named

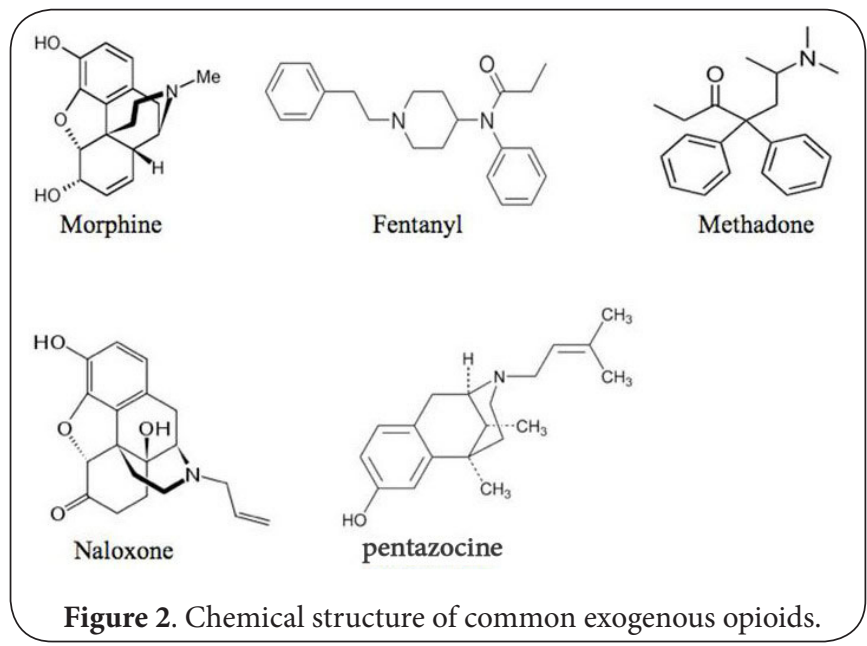

morphine, after Morpheus, the Greek god of dreams [11]. By the mid-nineteenth century, several other alkaloids derived from opium including codeine and papaverine were being used throughout the world in the field of medicine. Over the next 75 years, several additional synthetic opioid analgesics were developed including opioid agonists, antagonists, and mixed agonist-antagonist drugs that are commonly used today [12].

\section{Classification}

There are a variety of methods to classify the different opioids used in clinical practice. In terms of broad categories, there are either naturally occurring, semisynthetic, or synthetic opioids. Examples of naturally occurring opioids include morphine and codeine; semisynthetic opioids include hydromorphone, oxycodone, and hydrocodone; and synthetic opioids include fentanyl, methadone, and meperidine [11].

Opioids can also be divided by chemical structure (Figure 2). There are four main chemical classes of opioids:

1. Phenanthrenes are often considered the prototypical opioids and examples of intravenous formulations include morphine, hydromorphone, buprenorphine, nalbuphine, and butorphanol.

2. Phenylpiperidines encompass a variety of commonly used intravenous opioids including fentanyl, meperidine, alfentanil, and sufentanil.

3. Diphenylheptanes represent another chemical class of opioids, which includes methadone.

4. Benzomorphans are a small group that includes the agonist/antagonist pentazocine [1]. A detailed discus sion of the specific differences in chemical structure of each group is beyond the scope of this review.

Another method of opioid classification is based on the specific actions of the drug on specific opioid receptors, i.e. agonist, partial agonist, agonist/antagonist, and antagonist (please see next section). Opioid receptors function as $\mathrm{G}$ protein-coupled receptors, and are structurally characterized by 7 transmembrane domains (Figure 3 ). These receptors are 


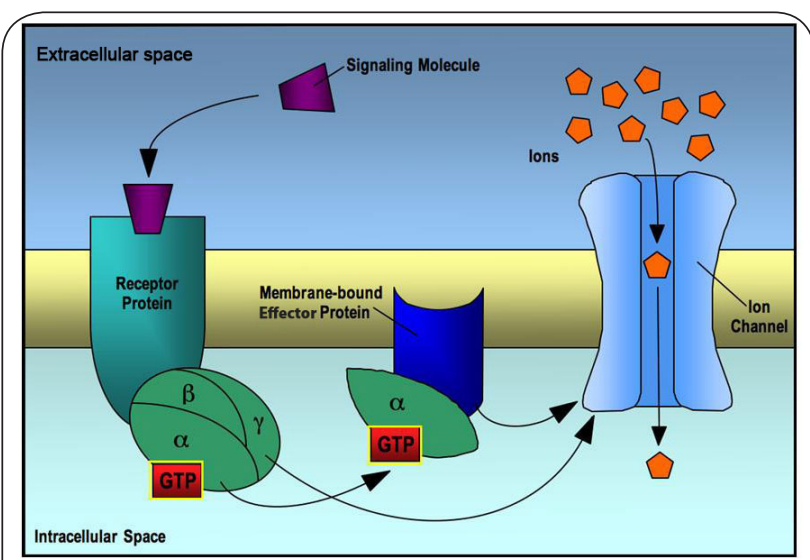

Figure 3. G-protein-coupled receptor. The signaling molecule binds to the membrane receptor protein, which then activates the G-protein heterotrimer. The alpha subunit of the heterotrimer, in conjunction with GTP, binds to and activates the membrane-bound effector protein. Both the effector protein and the $\beta \gamma$-subunit of the G-protein heterotrimer are capable of inducing opening of the ion channel.

located in all areas of the CNS involved in pain perception (brainstem, medial thalamus, spinal cord, hypothalamus, limbic system), and are also present throughout peripheral tissues [13]. There are three major classes of opioid receptors.

Mu $(\boldsymbol{\mu})$ : These receptors are located primarily in the brainstem as well as the medial thalamus, where they mediate supraspinal analgesia, respiratory depression, euphoria, sedation, decreased gastrointestinal motility, and physical dependence. There are two subtypes, known as Mu1 and Mu2. Mu1 receptors have higher affinity for morphine and mediate supraspinal analgesia and euphoria; Mu2 receptors have lower affinity for morphine and mediate spinal analgesia, respiratory depression, and dependence [1].

Kappa (K): These receptors are found in the limbic system, brain stem, and spinal cord. Their major effects include spinal analgesia, sedation, dyspnea and respiratory depression, dependence, and dysphoria [14].

Delta $(\boldsymbol{\delta})$ : These receptors are located throughout the brain and spinal cord and likely mediate spinal and supraspinal analgesia along with psychomimetic and dysphoric effects [14].

The majority of the traditional intravenous opioids used in the acute care setting are pure agonists, exerting their effect by directly stimulating opioid receptors. Partial agonists, such as buprenorphine, also stimulate opioid receptors directly, but are less efficacious than full agonists. Agonist/antagonists often have very poor efficacy at Mu opioid receptors (antagonist component), but have greater efficacy at other opioid receptors such as Kappa (agonist component). Examples of intravenous opioids that are considered agonist/antagonists include nalbuphine and butorphanol. Pure intravenous opioid antagonists act as competitive antagonists at all opioid receptor types with particularly high affinity for the $\mathrm{Mu}$ receptor, but lack any efficacy. The most common opioids in this group are naloxone and naltrexone [1].

\section{Mechanism of action}

The basic structure of morphine, the prototypical opioid, consists of five rings forming a T-shaped molecule (Figure 2). The important binding groups on morphine include the phenol, the aromatic ring, and the ionized amine, which are found in all opioid analgesics [1]. These groups appear to be essential to the analgesic activity of opioids, as substitution of the basic amino and phenol groups have been shown to result in a dramatic reduction in activity and analgesic effect [15].

The analgesic effects of intravenous opioids are the result of two related processes: a) direct inhibition of ascending transmission of nociceptive information from the dorsal horn of the spinal cord, and b) activation of descending pain control circuits from the midbrain to the dorsal horn of the spinal cord [11]. As mentioned previously, opioid receptors are G-protein-coupled receptors. After receptor activation, a portion of the $\mathrm{G}$ protein is released, which inhibits cyclic adenosine monophosphate (CAMP) and leads to alterations in gene transcription. More specifically, opioid agonists ultimately block the release of pain-related neurotransmitters such as glutamate and substance $P$ from nociceptive $C$-fibers and A-delta fibers, resulting in analgesia. Opioids (both endogenous and exogenous) will also activate presynaptic gamma-aminobutyric acid (GABA) receptors, which inhibits GABA release and allows increased dopaminergic transmission in the brain, ultimately causing a sensation of pleasure or euphoria [16]. Some opioids also act as N-Methyl-D-aspartic acid (NMDA) receptor antagonists, leading to analgesia by stimulation of the descending pain pathways from the brain stem via enhanced serotonin and noradrenaline transmission.

As discussed above, opioids are often classified based on the degree of agonist or antagonist activity at specific receptors. A full opioid agonist has both a high degree of affinity and efficacy at opioid receptors. Agonists represent the vast majority of intravenous opioids used in clinical practice, and are typically considered the most effective at producing analgesia, but also have the capability of producing the most side effects. A partial agonist has a high level of affinity but has significantly less efficacy at opioid receptors. These agents produce less pain relief and have a ceiling to their analgesic effect, so that escalating doses will not enhance analgesia but will instead, yield greater side effects. Given their high affinity at opioid receptors, they also have the potential to induce withdrawal in an opioid-dependent patient. These characteristics make partial agonists a reasonable choice for opioid abuse deterrence and maintenance therapy. An 


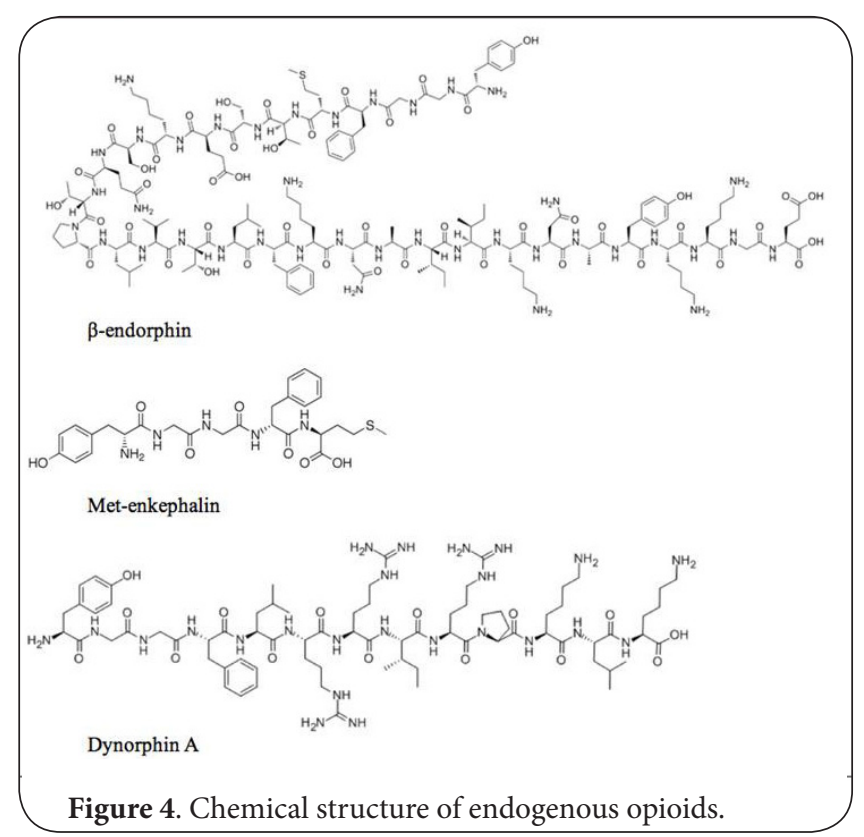

opioid agonist/antagonist has affinity but minimal efficacy at certain opioid receptors (antagonist component), but has greater efficacy at other receptor types (agonist component). These drugs can be used to treat pain, but like partial agonists, have a ceiling effect in terms of analgesia. They may also precipitate withdrawal in an opioid-dependent individual. A pure opioid antagonist has high affinity but no efficacy at opioid receptors. These agents function to reverse the undesirable effects of opioids, and are often combined with full or partial opioid agonists for opioid abuse deterrence and long-term maintenance therapy [1].

\section{Endogenous opioids}

There are three distinct families of endogenous opioid peptides located throughout the central and peripheral nervous systems and surrounding tissues (Figure 4). They function as neurotransmitters and likely play a role in hormone secretion, thermoregulation, and cardiovascular function. A basic understanding of this complex antinociceptive system involved in pain modulation is helpful in understanding the effects of intravenous opioid analgesics [13].

\section{Endorphins}

These compounds are derived from pro-opiomelanocortin and act at the Mu receptor (highest affinity for Mu1). They are synthesized in the hypothalamus as well as pituitary gland, and are released during pain, strenuous exercise, excitement, and orgasm. These peptides are often referred to as "natural pain killers" given their ability to create analgesia and a general sense of happiness. By stimulating the $\mathrm{Mu}$ receptor, they inhibit the release of GABA, which normally inhibits dopamine release, therefore resulting in enhanced dopamine emission [17]. Endorphins also likely play a major role in the pain-mediating effects of acupuncture, massage, hydrotherapy, and TENS therapy, as research has shown that these popular treatment modalities consistently stimulate the body's production of these compounds $[\mathbf{1 8}, \mathbf{1 9}]$.

\section{Enkephalins}

These endogenous peptide neurotransmitters are derived from pro-enkephalin and act mainly at the Delta receptor. They are located throughout the brain and spinal cord and are involved in regulating nociception in the body. Enkephalins inhibit neurotransmitters in the pathway for pain perception, thereby reducing the emotional and physical impact of pain [13]. Interestingly, enkephalins are also found in the $\mathrm{Gl}$ tract where they affect pancreatic enzyme secretion and metabolism of carbohydrates [13].

\section{Dynorphins}

These peptides are synthesized from the precursor prodynorphin and exert their effects mainly through the Kappa receptor. Located throughout the CNS, with the highest concentrations found in the brainstem, hypothalamus, and spinal cord, they perform a variety of physiological actions. Their primary functions are to modulate the pain response, control appetite and weight, maintain circadian rhythm, and regulate body temperature [13]. Dynorphins have been shown to play a major role in cocaine addiction, as repeated exposure to the illicit drug results in increased dynorphin concentrations in the brain [20]. They have also been linked to stress-induced depression and drug-seeking behavior in rats [21]. Drugs that block the effects of dynorphins are currently being studied as possible treatments for depression related to drug addiction [21].

\section{Exogenous opioids \\ Morphine}

Morphine is undoubtedly the prototypical opioid. It is predominantly a Mu receptor agonist and is a phenanthrene derivative. Morphine produces analgesia, euphoria, and a sensation of warmth in a dose-dependent fashion. Despite persistent pain, even low doses of morphine increase the threshold to pain and modify the perception of noxious stimuli. It is most useful in treatment of continuous, dull pain, and is effective against pain arising from visceral organs as well as discomfort from skeletal muscles, joints, and bone. When administered intravenously, the peak effect requires 15 to 30 minutes and the duration of action is approximately 4 hours [12]. The elimination half-life is 1.7-3.3 hours and the volume of distribution is $4-5 \mathrm{~L} / \mathrm{kg}$. Morphine has poor lipid solubility and is metabolized by conjugation with glucuronic acid in the liver and kidneys. The primary metabolites are morphine-3-glucuronide (75-85\%), which is pharmacologically inactive, and morphine-6-glucuronide (5-10\%) which acts on Mu receptors to produce analgesia and respiratory depres- 
Table 1. Equipotent intravenous dosages of common opioids. To convert $\mathrm{mg}$ to $\mathrm{mcg}$, multiply by $1000(0.02 \mathrm{mg}=20 \mathrm{mcg})$.

\begin{tabular}{lc}
\hline Drug & IV Dose $(\mathrm{mg})$ \\
\hline Morphine & 2 \\
Hydromorphone & 0.3 \\
Fentanyl & 0.02 \\
Meperidine & 15 \\
Methadone & 0.4 \\
Buprenorphine & 0.1 \\
Butorphanol & 0.4 \\
Nalbuphine & 2 \\
\hline
\end{tabular}

Table 2. Adverse effects associated with the use of opioids.

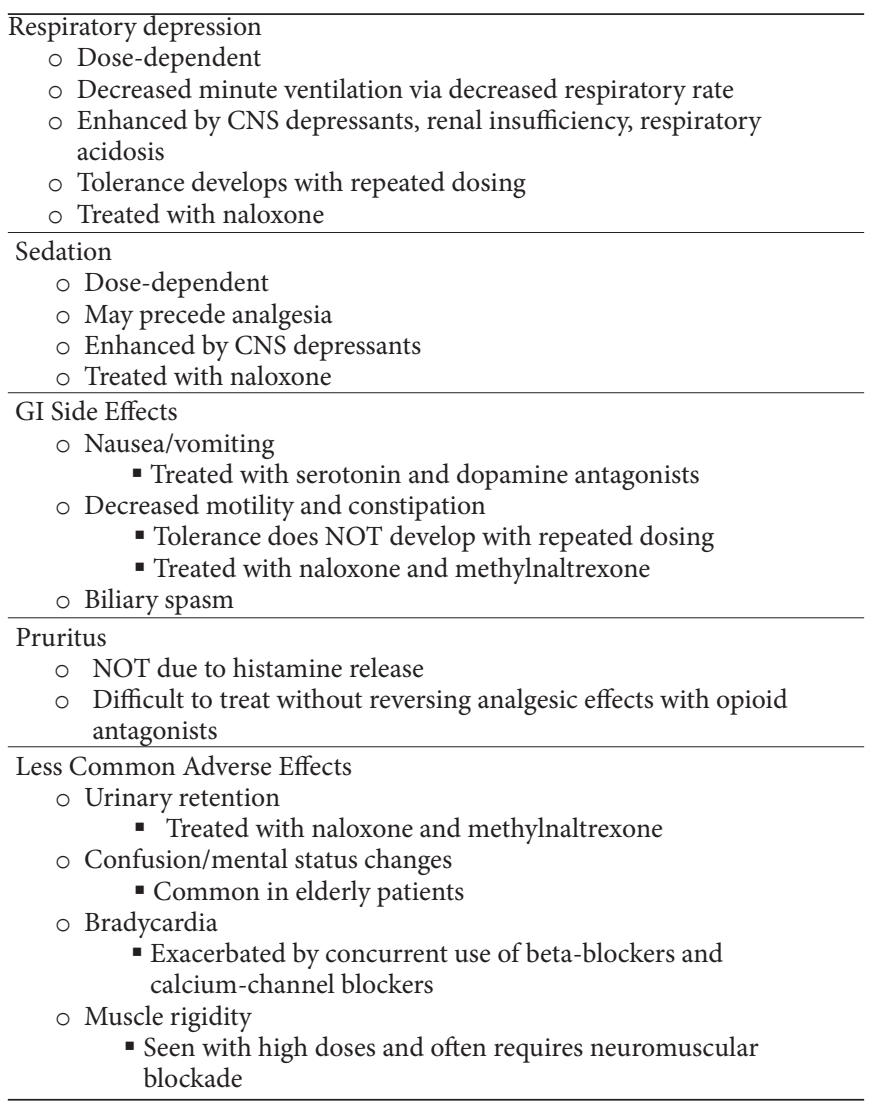

sion [22]. Morphine is primarily excreted in urine (90\%), and therefore accumulation of metabolites resulting in prolonged drug effects are often seen in patients with renal failure. A detailed discussion of therapeutic, toxic, and fatal levels of morphine and the other common opioids is beyond the scope of this review, but are important considerations for clinicians prescribing these potent drugs [46].

Common side effects with all opioids include nausea, sedation, inability to concentrate, dry mouth, pruritus, constipation, and respiratory depression (Table 2). One specific adverse effect, seen most commonly with morphine, is his- tamine release. This is relatively common, especially when the drug is administered as a rapid intravenous bolus, and can lead to hypotension, bronchospasm, and facial flushing [12]. Although morphine has commonly been used to treat chest pain in patients suspected of having acute coronary syndrome, data suggests that its use in patients with unstable angina and non-ST segment elevation myocardial infarction may actually increase mortality and therefore should be used cautiously if at all in this group [40]. Typical dosing of parenteral morphine is $0.05-0.1 \mathrm{mg} / \mathrm{kg}$ (or 2-10 $\mathrm{mg}$ in adults) as an initial loading dose, followed by repeat dosing every 2-6 hours as needed (Please refer to Table 1 for equipotent doses of common opioids). When administered via patientcontrolled analgesia (PCA), typical demand doses are 1-2 mg with a 10-15 minute lockout to facilitate adequate time for the bolus dose to start taking effect. The 1-hour PCA limit programmed for an opioid naïve patients with moderate pain is typically 6-8 $\mathrm{mg}$. These are very basic recommendations, and adjustments are often required based on multiple factors including patient age, prior opioid use, and patient-to-patient (genetic) variability. Moreover, it is essential to observe an individual patient's clinical response to a given dose when using a PCA so that the demand dose can be appropriately adjusted to prevent over-administration of narcotic. In patients with renal failure, doses should be decreased 10-50\% based on the severity of the disease. Decreased dosing is also recommended in patients with severe liver disease and in the elderly. Reduced clearance of morphine has also been shown in patients suffering from acute trauma, likely secondary to reduced hepatic blood flow and altered protein binding, which can potentially lead to accumulation and toxicity in these patients in the intensive care setting [41].

\section{Hydromorphone}

Another very common intravenous opioid used in the inpatient setting is the semi-synthetic opioid agonist hydromorphone, a morphine derivative. It acts primarily on Mu receptors and is roughly 5-7 times as potent as morphine. It has similar effects as morphine, but may produce less sedation and more euphoria. After intravenous administration, the peak effect of hydromorphone occurs somewhat more quickly than Morphine, in approximately 5-10 minutes. The duration of action is slightly less, with effects typically lasting 3-4 hours. The elimination half-life is approximately 2.3 hours and the volume of distribution is $2.5-3.5 \mathrm{~L} / \mathrm{kg}$. Hydromorphone is metabolized in the liver, with the primary metabolite being hydromorphone-3-glucuronide which has no analgesic effect [23]. There is some recent evidence to suggest that hydromorphone may be a more effective analgesic than morphine in treating acute pain [24]. A 2011 meta-analysis by Felden et al., compared the clinical effects of morphine and hydromorphone in 8 studies published through 2009 ( $n=1004$ patients). Hydromorphone provided significantly better analgesia than morphine for acute pain, with the 
advantage of hydromorphone over morphine being more pronounced in the higher quality studies. There were no significant differences in terms of adverse effects between the two drugs [24].

Dosing of intravenous hydromorphone in the inpatient setting is also highly variable depending on the patient. A typical loading dose is $0.2-0.6 \mathrm{mg}$, with repeat boluses every 2-3 hours as needed. Like morphine, it is important to wait an adequate length of time prior to re-dosing, given the time required for peak effect. When administered via PCA, a common starting demand dose is $0.2-0.4 \mathrm{mg}$ with a lockout of 6-10 minutes and a 1-hour limit of 1.2-1.5 mg. Although there are no specific recommendations, it is common to decrease the loading dose and titrate slowly in the elderly and in patients with severe liver or kidney disease.

\section{Fentanyl}

Fentanyl is a synthetic Mu receptor agonist that is $80-100$ times more potent than morphine. It is structurally similar to meperidine, and is highly lipophilic. This results in rapid passage across the blood-brain barrier, therefore leading to quick onset after intravenous administration (2-3 minutes) and short duration of action (45-60 minutes) from rapid redistribution to inactive tissues. However, after continuous infusions, or even multiple consecutive intravenous boluses, a saturation of these inactive tissue sites may lead to a "sink" phenomena, where the depot of fentanyl leaches back into the systemic circulation after the initial intravenous doses are stopped. As such, the effects of fentanyl are often prolonged in the elderly. The elimination half-life is approximately 3.5 hours and the volume of distribution is 4-6 L/ kg. Fentanyl is metabolized primarily in the liver to the inactive metabolites norfentanyl, hydroxyproprionyl-fentanyl, and hydroxyproprionyl-norfentanyl, which are all excreted in the urine [12].

Standard doses of intravenous fentanyl for analgesia are $1-2 \mathrm{mcg} / \mathrm{kg}(50-100 \mathrm{mcg}$ in the average adult) as a loading dose. Again, doses should be decreased and titrated appropriately in the elderly; no specific adjustments are needed in patients with renal or liver disease. Given its fast onset and short duration of action, fentanyl is typically more useful in situations where brief but intense periods of pain relief are required such as procedural analgesia. Fentanyl is also very effective for rapid relief of severe pain until a longer acting agent has time to take effect; moreover, given that fentanyl has been shown to produce fewer side effects (e.g. sedation, nausea/vomiting, urinary retention, pruritus) than morphine and hydromorphone, it may actually be a preferable drug for patient controlled analgesia in the acute care setting [25].

\section{Meperidine}

Meperidine is a weak synthetic Mu and Kappa receptor agonist, which is about one-tenth as potent as morphine. It shares structural similarities with both local anesthetics and atropine, but its principal pharmacologic effects are consistent with opioids. Meperidine may produce more euphoria than other opioids, which can result in abuse and drug-seeking behavior in susceptible patients. When administered intravenously, the time to onset is 5-10 minutes and the duration of action is 2-4 hours [12]. The elimination half-life is 3-5 hours and the volume of distribution is roughly $4 \mathrm{~L} / \mathrm{kg}$. Meperidine is extensively metabolized in the liver by glucuronidation to normeperidine, which is in turn, renally excreted. In patients with impaired renal function, normeperidine can accumulate, and given its long half-life, can result in CNS toxicity with symptoms including tremors, myoclonus, and seizures [26]. Typical side effects of meperidine resemble those of morphine, although hypotension may be more profound after a bolus dose of meperidine. Increased heart rate after intravenous administration is not uncommon, which is not surprising given the similarities in its structure to atropine. Administration of meperidine in patients on monoamine oxidase inhibitors (MAOIs) can precipitate serotonin syndrome, which can lead to profound autonomic instability (e.g. hypertension, tachycardia, diaphoresis, hyperthermia, agitation, coma, seizures, and even death).

Typical doses of intravenous meperidine are $25-50 \mathrm{mg}$ $(0.5-1 \mathrm{mg} / \mathrm{kg})$ as a loading dose, with repeat dosing every 2-3 hours as needed. In patients with kidney disease, this dose should be decreased $25-50 \%$ depending on the severity of renal dysfunction; decreased dosing and slow titration is also recommended in the elderly. Given the concern over normeperidine accumulation and toxicity, meperidine is rarely administered via PCA delivery systems [27]. In addition to being used for managing pain in the in patient setting, meperidine is also useful for treating post-operative shivering.

\section{Methadone}

Methadone is a unique synthetic Mu receptor agonist that is very commonly used in the management of chronic pain and opioid addiction, but can also be effective in treating acute pain when used appropriately. In addition to its effects on opioid receptors, methadone is also an NMDA receptor antagonist, adding to its analgesic properties. The elimination half-life is highly variable, ranging from 8-60 hours, and the volume of distribution is $5-6 \mathrm{~L} / \mathrm{kg}$. It is metabolized in the liver to inactive substances and is excreted by the kidneys [1]. It is considered a longer-acting opioid, as single doses of intravenous methadone can produce analgesia for 4-8 hours. This necessitates very cautious administration as repeated dosing within short intervals can result in drug accumulation and toxicity. When methadone is administered repeatedly (i.e. patients with chronic pain or prior heroin abuse), it accumulates in tissue and equilibrates with drug circulating in plasma sustaining an effective plasma level for up to 24 hours. This reservoir of drug explains why patients on maintenance therapy require only once daily dosing, while in the acute setting the effects are only sustained for approximately 6 hours [43]. Methadone produces very similar analgesic effects as other commonly 
used opioids, but it lacks the euphoric effects of these drugs, which may be beneficial in certain patient populations. In terms of adverse effects, methadone is unique, as it has the potential to cause QT interval prolongation and Torsades de Pointes in susceptible patients [28].

Methadone is essentially equipotent to morphine. Common intravenous dosing is $2.5-10 \mathrm{mg}$ as a loading dose, with cautious repeat dosing every $8-12$ hours as needed. Time to full analgesic (and respiratory) effect is variable and may take up to 2-3 days, so vigilance is paramount to the successful use of methadone. Though not a common first-line choice for treatment of pain in the inpatient setting, intravenous methadone can be very effective in managing patients with true morphine allergies. In patients predicted to have longterm opioid requirements, intravenous methadone can easily be transitioned to the oral formulation. It is also often helpful in managing patients with a history of prolonged opioid use and/or high opioid tolerance, as the NMDA effects may be beneficial in such challenging patients [12].

\section{Buprenorphine}

Buprenorphine is an opioid agonist-antagonist that is commonly used as an oral formulation in the management of chronic pain and opioid addiction. It acts as a partial agonist at the Mu and Delta receptors, and as a competitive antagonist at the Kappa receptor. Buprenorphine has high analgesic potency, as it is roughly 20-40 times more portent than morphine. After intravenous administration, peak effect may take up to 60 minutes, and the duration of action is often greater than 8 hours. It is metabolized by the liver and is subsequently excreted mainly in bile. Given its high affinity for Mu receptors, buprenorphine is relatively resistant to antagonism with naloxone, requiring higher doses for successful reversal [29]. Although not commonly used in the acute care setting for pain management, buprenorphine can be very effective in relieving moderate to severe pain. Side effects are similar to other opioids, but it is important to remember that as a result of its antagonist properties, buprenorphine can precipitate withdrawal symptoms in patients who are physically dependent on other commonly used opioids [12].

Standard dosing for intravenous buprenorphine is 150300 mcg every 6-8 hours as needed, titrated to effect. This dose should be decreased by roughly $50 \%$ in the elderly, or in patients with significant respiratory disease, given the challenges of reversing undesired side effects such as respiratory depression. The elimination half-life ranges from 20-60 hours and the volume of distribution is $3-5 \mathrm{~L} / \mathrm{kg}$. As the drug is hepatically metabolized and cleared, dosing should also be decreased in patients with severe liver disease. Although intravenous buprenorphine is not a traditional opioid used for pain control, it may be beneficial in certain groups of patients in the inpatient setting, such as current opioid abusers or patients who are on long-term maintenance programs for past opioid addiction.

\section{Butorphanol}

Butorphanol is a synthetic opioid agonist-antagonist, which has low affinity for Mu receptors (where it has an antagonist effect) and moderate affinity for Kappa receptors (where it has an agonist effect). Stimulation of the Kappa receptors produces analgesia, but can also cause dysphoria in higher doses, making butorphanol advantageous in comparison to other opioids from the point of abuse potential [1]. Although butorphanol is most commonly used as an intranasal formulation for the treatment of migraines, the intravenous formulation can also be effective in the treatment of moderate to severe pain. The onset of action after intravenous administration is 10-15 minutes and the duration of action is 3-4 hours. The elimination half-life is 4.5-5 hours and the volume of distribution is variable, ranging from 4-12 L/kg. In the liver, butorphanol is converted to hydroxybutorphanol, an inactive metabolite that is predominantly eliminated in bile and to a lesser extent, in urine. Side effects are similar to those seen with most other opioids, although it is not uncommon to see elevations in systemic and pulmonary pressures and cardiac output after administration, and thus it should be used cautiously in patients with underlying cardiac disease [11].

Typical dosing of intravenous butorphanol is $0.5-2 \mathrm{mg}$ as a loading dose and re-administration may be every 3-4 hours as needed. In patients with severe liver or kidney disease, dosing frequency should only be every 6 hours, given the potential for accumulation and drug toxicity. Intravenous butorphanol is most commonly used in the post-operative setting for pain relief, but is also commonly used for pain control in women during labor. It may be effective in treating pain in patients who have abused opioids in the past as its agonist activity is primarily directed towards the Kappa receptor.

\section{Nalbuphine}

Nalbuphine is an opioid agonist-antagonist that is structurally similar to naloxone. It is equipotent to morphine in terms of analgesia. Nalbuphine functions as an antagonist at Mu receptors and as an agonist at Kappa receptors. When administered intravenously, the onset of effect is within 5-10 minutes and its duration of action is 3-6 hours [11]. The elimination halflife is approximately 5 hours, and the volume of distribution is $4-5 \mathrm{~L} / \mathrm{kg}$. It is inactivated in the liver and renally excreted. Sedation is the most common side effect, and nalbuphine appears to produce less dysphoria compared to other opioid agonist-antagonists. It does not increase blood pressure or cardiac output. Because of its strong antagonist effects on $\mathrm{Mu}$ receptors, the subsequent use of opioid Mu receptor agonists after administration of nalbuphine may prove ineffective at providing adequate analgesia [12]. On the other hand, because of its unique properties, nalbuphine is effective at reversing both respiratory depression and pruritus produced by opioid Mu receptor agonists, while still maintaining analgesia [30].

A standard loading dose of intravenous nalbuphine is 5-10 
mg titrated to effect, which can be repeated every 3-6 hours as needed. Decreased dosing in the elderly and in patients with severe liver disease is recommended. Caution should be exercised when administering it to patients with severe pain, as the antagonist effects may limit the ability to subsequently use opioid Mu receptor agonists, if needed. Nalbuphine may be a good choice for the treatment of pain in patients who are susceptible to severe respiratory depression with standard opioids, or in those prone to the common side effects of opioids such as pruritus, nausea, and vomiting.

\section{Naloxone}

Naloxone is a nonselective opioid antagonist, affecting all three types of opioid receptors. It is clinically used to treat the adverse effects associated with opioids, most commonly respiratory depression, but also pruritus and gastrointestinal effects (constipation and/or opioid-induced ileus). Unfortunately, naloxone also reverses the most desired effect of opioids, i.e. analgesia. As such, it must be used with caution and titrated to the appropriate effect. The onset of action is extremely rapid (within 1-2 minutes) and the duration of action is 30-45 minutes. The elimination half-life is 1-1.5 hours and the volume of distribution is $2-5 \mathrm{~L} / \mathrm{kg}$. It is metabolized in the liver to naloxone-3-glucuronide, which is renally eliminated [12]. Cardiovascular stimulation from the acute perception of severe pain and associated sympathetic nervous system activation after administration of high doses of naloxone can result in tachycardia, hypertension, pulmonary edema, and cardiac dysrhythmias [31].

The recommended starting dose for intravenous naloxone to reverse the effects of opioids is $1-4 \mathrm{mcg} / \mathrm{kg}$, slowly titrated to desired effect, with repeat dosing every $30-45$ minutes as needed. Given the short duration of action of Naloxone and the often long half-lives of common opioids used for pain control, it is not uncommon to see recurrence of respiratory depression ("renarcotization") or other side effects, requiring either repeated doses or a naloxone infusion (e.g. $5 \mathrm{mcg} / \mathrm{kg} /$ hr) [11].

\section{Methods of adiministration}

The options for intravenous administration of opioids include intermittent bolus dosing, patient controlled analgesia (PCA) dosing, and continuous infusions. Continuous infusions of opioids are rarely a viable option for patients in the acute care setting given the inherent risks of overdose and severe side effects; however, this method of opioid administration may be useful in the occasional patient who is highly opioid tolerant and is under the direct supervision of a pain medicine physician. With this in mind, the two viable options for intravenous administration of opioids include intermittent bolus (PRN) and/or patient controlled analgesia (PCA) dosing. With PRN dosing, patients receive medication either when their bedside nurse deems the pain to be severe enough or if the patient requests it (assuming that the maximum al- lowable amount has not been exceeded). With PCA dosing, a sophisticated microprocessor-controlled infusion pump delivers a preprogrammed dose of opioid when the patient presses a demand button. A set lock-out period takes effect after receiving a demand dose and a total one-hour limit is programmed, both to allow for titration to the desired effect and to prevent accidental overdose. The general idea behind PCA is that small, frequent boluses of intravenous opioids provide better pain relief than larger boluses administered less often [32].

A 2011 Cochrane Review article assessed patient controlled opioid analgesia vs. conventional opioid analgesia in the management of postoperative pain [33]. Fifty-five randomized, controlled trials were included in the meta-analysis, which assessed pain management in over 3800 patients. PCAs were associated with better pain control and greater patient satisfaction vs. conventional "as needed" analgesia. And while patients using PCA consumed a higher amount of intravenous opioids, they reported similar levels of side effects and had equivalent lengths of stay as patients receiving PRN dosing of intravenous opioids. The conclusion of the review, which was in concordance with several prior meta-analyses, was that PCA provides superior pain relief when compared with conventional analgesia and that patients report greater satisfaction and prefer PCA when receiving intravenous opioids for postoperative pain [33].

Despite the effectiveness of PCA for intravenous opioid therapy in the acute care setting, there are documented risks associated with its use [34]. Both human and equipment errors are possible, including administration of the wrong analgesic, pump misprogramming, equipment malfunction, false triggering, and/or accumulation of a drug in the dead-space on intravenous line tubing. Because of these potentially life-threatening errors, Tran et al., assessed the impact of implanting "smart" PCAs (computerized pumps with medication safety enhancements - particularly related to operator errors during administration) in both, medical and surgical patients, over a 6-month period [34]. The use of smart pumps was credited with preventing 159 potential errors during this time, with the nursing staff expressing strong positive attitudes towards the utility of these pumps to decrease the occurrence of PCA-related errors and improve patient safety [34]. Though isolated to a single university-based healthcare system, this data suggests that smart PCA pumps are a safe and effective way to manage pain in a mixed acute care patient cohort.

A simple algorithm for managing an intravenous PCA system should start with an initial loading dose of the selected opioid (See Table 3), which may be administered every 5-10 minutes until the pain score is less than 4 or respiratory rate is less than 12 breaths/minute. If the patient subsequently complains of inadequate pain relief, the administration of a bolus dose and increase in the demand dose should be considered, after making sure that the patient has been 
Table 3. Common Initial PCA Regimens for Opioid-Naïve Patients. Adapted with permission from "Patient-Controlled Analgesia" [32]. Continuous basal infusions are NOT recommended for opioid-naïve patients.

\begin{tabular}{lcc}
\hline Opioid & Demand Dose & Lockout (min) \\
\hline Morphine & $1-2 \mathrm{mg}$ & $6-10$ \\
Hydromorphone & $0.2-0.4 \mathrm{mg}$ & $6-10$ \\
Fentanyl & $20-50 \mathrm{mcg}$ & $5-10$ \\
Meperidine & $10-20 \mathrm{mg}$ & $6-10$ \\
\hline
\end{tabular}

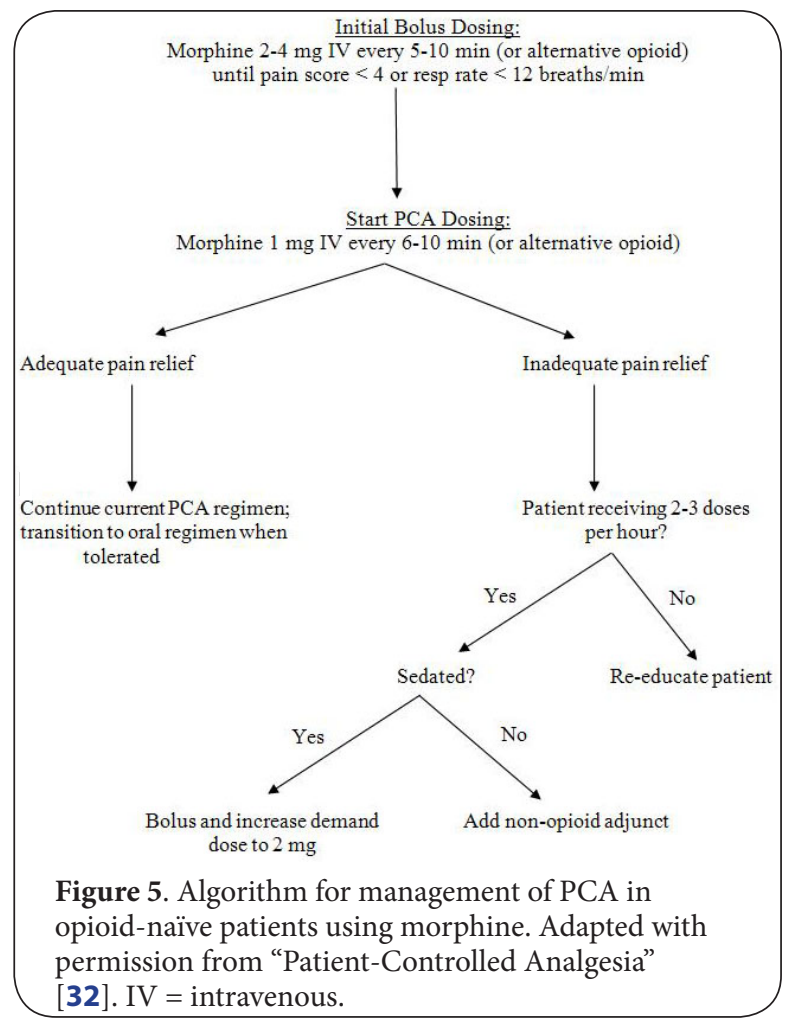

appropriately pushing the PCA button and that the pump is functioning properly (See Figure 5). Demand dose escalation should always be thoroughly discussed with the patient, as he/she must consider the potential for worsening side effects with higher doses of opioids. The addition of a non-opioid adjunct, such as a nonsteroidal anti-inflammatory drug or acetaminophen, may help decrease opioid requirements [32]. As mentioned previously, continuous basal infusions should be used cautiously, if at all, especially in opioid-naïve patients; if deemed necessary, a pain management physician should be consulted given the increased potential for lifethreatening consequences. Despite these techniques, there are the occasional patients with difficult to manage acute pain who may require more aggressive therapy. Advances in assessing opioid drug levels in plasma combined with individual pharmacogenomics through drug genotyping may lead to more optimal pain control and less adverse effects in these challenging patients [42]. Obesity is an increasingly
Table 4. Drugs interactions with methadone.

\begin{tabular}{ll}
\hline $\begin{array}{l}\text { CYP3A4 Inducers } \\
\text { (decreased effect) }\end{array}$ & $\begin{array}{l}\text { CYP3A4 Inhibitors } \\
\text { (increased effect) }\end{array}$ \\
\hline Carbamazepine & Ciprofloxacin \\
Rifampin & Anti-retroviral agents \\
Phenytoin & Calcium channel blockers \\
Phenobarbital & Anti-fungal agents \\
Dexamethasone & Macrolide antibiotics \\
Nafcillin & Isoniazid \\
\hline
\end{tabular}

common problem in the US today, and these patients present unique obstacles in pain management. They often require alterations in doses of opioids given variations in lean body mass, volume of distribution, and clearance of drugs. The effects of respiratory depression and hypoventilation on these patients can be more profound, and this requires close attention when caring for such patients [44]. The management of opioid-tolerant patients frequently requires higher initial bolus and demand dosages along with continuous infusions, and is beyond the scope of this review.

\section{Drug interaction}

Drug interactions are an important concept as patients tend to be prescribed multiple agents at any one time [35]. Polypharmacy, or the use of multiple medications by a patient, is an increasingly common problem in the US, especially in the elderly population, and puts patients at higher risk for adverse drug reactions as well as drug-drug interactions [35]. A drug-drug interaction is said to occur when either the quantity or the effect of a drug is altered by the concurrent administration of another drug(s) [36]. One of the most dangerous interactions is that of MAOls and meperidine, which can induce serotonin syndrome [37]. Tricyclic antidepressants (e.g. nortriptyline, amitriptyline) may inhibit morphine glucuronidation and result in elevated opioid plasma levels [1]. Erythromycin can also prolong opioid effects by inhibition of the oxidizing activity of cytochrome P450 [11].

Methadone has multiple drug interactions that must be considered (See Table 4). Carbamazepine, rifampin, phenytoin, phenobarbital, oral hypoglycemic agents, and other CYP3A4 inducers all enhance methadone metabolism, leading to decreased blood levels. This results in decreased efficacy of methadone and possible withdrawal symptoms [38]. On the other hand, co-administration of CYP3A4 inhibitors such as ciprofloxacin, antiretroviral agents, calcium channel blockers, antifungals, and macrolide antibiotics can reduce the metabolism of methadone, leading to elevated plasma levels and possible toxicity [12].

\section{Conclusion}

Administration of intravenous opioids is an essential component of acute pain management in the inpatient setting. Opioids can be classified in a variety of ways including 
chemical structure and specific mechanism of action. An understanding of opioid pharmacology - including receptor type, endogenous opioids, and mechanism of action - is critical to the safe and responsible use of these potent drugs. Moreover, an appreciation of the clinical pharmacokinetics of these complex drugs - including elimination half-life, volume of distribution, and metabolism - is also essential to providing effective pain management in the acute care setting. A variety of agents can be administered intravenously, and each differs in its clinical effects, potential for toxicity, and side effect profile. A thorough understanding of these factors is essential when administering these drugs, especially in specific patient populations, including the elderly and those with significant comorbidities. Drug-drug interactions can be an issue when prescribing opioids, most notably in complex patients with co-existing kidney or liver disease.

\section{Competing interests}

The authors declare that they have no competing interests

Publication history

Received: 26-July-2012 Revised: 01-Jan-2013

Accepted: 02-Jan-2013 Published: 08-Jan-2013

\section{References}

1. Trescot $A M$ M, Datta S, Lee $M$ and Hansen H: Opioid pharmacology. Pain Physician 2008, 11:S133-53. | Article | PubMed

2. Bohnert A S, Fudalej $S$ and Ilgen M A: Increasing poisoning mortality rates in the United States, 1999-2006. Public Health Rep 2010, 125:5427. | PubMed Abstract | PubMed Full Text

3. Dix P, Sandhar B, Murdoch J and MacIntyre P A: Pain on medical wards in a district general hospital. Br J Anaesth 2004, 92:235-7. | Article | PubMed

4. Kirsch $B, H$ Berdine and $D$ Zablotski. Management strategy: identifying pain as the fifth vital sign. VHSJ 2000; 49-59. I Article

5. Maga J. The Safety and Financial Implications of an Acute Pain Service. American Society of Anesthesiologists Newsletter 2012, 76:14-17. | Article

6. Coolen $\mathrm{P}$, Best $\mathrm{S}$. Overdose deaths involving prescription opioids among Medicaid enrollees - Washington, 2004-2007. MMWR Morb Mortal Wkly Rep 2009, 58:1171-5. | Article | PubMed

7. Apfelbaum J L, Chen C, Mehta S S and Gan T J: Postoperative pain experience: results from a national survey suggest postoperative pain continues to be undermanaged. Anesth Analg 2003, 97:534-40. I Article I PubMed

8. Helfand $M$ and Freeman $M$ : Assessment and management of acute pain in adult medical inpatients: a systematic review. Pain Med 2009, 10:1183-99. | Article | PubMed

9. Murnion $B$ P, Gnjidic D and Hilmer S N: Prescription and administration of opioids to hospital in-patients, and barriers to effective use. Pain Med 2010, 11:58-66. | Article | PubMed

10. McCaffery M, Ferrell B R and Pasero C: Nurses' personal opinions about patients' pain and their effect on recorded assessments and titration of opioid doses. Pain Manag Nurs 2000, 1:79-87. | Article | PubMed

11. Fukuda K. Opioids. Miller's Anesthesia, $7^{\text {th }}$ edition. Churchill Livingstone, Philadelphia 2010, pp 769-824.

12. Stoelting RK and Hillier SC. Opioid agonists and antagonists. Pharmacology \& Physiology in Anesthetic Practice, $4^{\text {th }}$ edition. Lippincott Williams \& Wilkins, Philadelphia 2006, pp 87-126.

13. Koneru A, Satyanarayana S and Rizwan S. Endogenous opioids: Their physiological role and receptors. Global Journal of Pharmacology 2009, 3:149-153. I PDF

14. Snyder S H and Pasternak G W: Historical review: Opioid receptors. Trends Pharmacol Sci 2003, 24:198-205. | Article | PubMed

15. Thorpe $D \mathrm{H}$ : Opiate structure and activity--a guide to understanding the receptor. Anesth Analg 1984, 63:143-51. | Article I PubMed

16. McCleane $\mathrm{G}$ and Smith $\mathrm{H}$ S: Opioids for persistent noncancer pain. Med Clin North Am 2007, 91:177-97. | Article | PubMed

17. Dalayeun J F, Nores J M and Bergal S: Physiology of beta-endorphins. A close-up view and a review of the literature. Biomed Pharmacother 1993, 47:311-20. | PubMed

18. Bender T, Nagy G, Barna I, Tefner I, Kadas E and Geher P: The effect of physical therapy on beta-endorphin levels. Eur J Appl Physiol 2007, 100:371-82. | Article | PubMed

19. Liu J L, Chen S P, Gao Y H, Meng F Y and Wang J Y: [Observation on the analgesic effect of repeated electroacupuncture and its relation to changes of plasma beta-EP, ACTH and COR levels]. Zhen Ci Yan Jiu 2007, 32:306-12. | PubMed

20. Wang B, Shaham Y, Zitzman D, Azari S, Wise R A and You Z B: Cocaine experience establishes control of midbrain glutamate and dopamine by corticotropin-releasing factor: a role in stress-induced relapse to drug seeking. J Neurosci 2005, 25:5389-96. | Article | PubMed

21. Shirayama Y, Ishida H, Iwata M, Hazama G I, Kawahara R and Duman R S: Stress increases dynorphin immunoreactivity in limbic brain regions and dynorphin antagonism produces antidepressant-like effects. J Neurochem 2004, 90:1258-68. | Article | PubMed

22. Romberg R, Olofsen E, Sarton E, Teppema L and Dahan A: Pharmacodynamic effect of morphine-6-glucuronide versus morphine on hypoxic and hypercapnic breathing in healthy volunteers. Anesthesiology 2003, 99:788-98. I Article | PubMed

23. Murray A and Hagen N A: Hydromorphone. J Pain Symptom Manage 2005, 29:S57-66. | Article | PubMed

24. Felden L, Walter C, Harder S, Treede R D, Kayser H, Drover $D$, Geisslinger $G$ and Lotsch J: Comparative clinical effects of hydromorphone and morphine: a meta-analysis. Br J Anaesth 2011, 107:319-28. | Article | PubMed

25. Hutchison RW, Eun Hae Chon, William F. Tucker, Richard Gilder, Janet Moss and Phyllis Daniel. A Comparison of a Fentanyl, Morphine, and Hydromorphone Patient-Controlled Intravenous Delivery for Acute Postoperative Analgesia: A Multicenter Study of Opioid-Induced Adverse Reactions. Hospital Pharmacy 2006, 41:659-663. | Article

26. Yeh $\mathrm{S} Y$, Krebs $\mathrm{H} A$ and Changchit $\mathrm{A}$ : Urinary excretion of meperidine and its metabolites. J Pharm Sci 1981, 70:867-70. | Article | PubMed

27. Stone $P A$, Macintyre $P E$ and Jarvis D A: Norpethidine toxicity and patient controlled analgesia. Br J Anaesth 1993, 71:738-40. | Article I PubMed

28. Krantz M J, Lewkowiez L, Hays H, Woodroffe M A, Robertson A D and Mehler P S: Torsade de pointes associated with very-high-dose methadone. Ann Intern Med 2002, 137:501-4. | Article | PubMed

29. Gal T J: Naloxone reversal of buprenorphine-induced respiratory depression. Clin Pharmacol Ther 1989, 45:66-71. | Article | PubMed

30. Bailey P L, Clark N J, Pace N L, Stanley T H, East K A, van Vreeswijk $\mathrm{H}$, van de Pol P, Clissold M A and Rozendaal W: Antagonism of postoperative opioid-induced respiratory depression: nalbuphine versus naloxone. Anesth Analg 1987, 66:1109-14. | Article | PubMed

31. Partridge $B L$ and Ward C F: Pulmonary edema following low-dose naloxone administration. Anesthesiology 1986, 65:709-10. | Article | PubMed

32. Grass J A: Patient-controlled analgesia. Anesth Analg 2005, 101:S4461. | Article | PubMed

33. Hudcova J, McNicol E, Quah C, Lau J and Carr D B: Patient controlled opioid analgesia versus conventional opioid analgesia for postoperative pain. Cochrane Database Syst Rev 2006, CD003348. | Article | PubMed

34. Tran M, Ciarkowski S, Wagner D and Stevenson J G: A case study on the safety impact of implementing smart patient-controlled 
Mckeen et al. Journal of Anesthesiology and Clinical Science 2013, http://www.hoajonline.com/journals/pdf/2049-9752-2-1.pdf

analgesic pumps at a tertiary care academic medical center. Jt Comm J Qual Patient Saf 2012, 38:112-9. | Article | PubMed

35. Fulton $M M$ and Allen $E$ R: Polypharmacy in the elderly: a literature review. J Am Acad Nurse Pract 2005, 17:123-32. | Article | PubMed

36. Jackson KC and Lipman AG. Opioid analgesics. Practical Pain Management, $\mathbf{3}^{\text {rd }}$ edition. Lippincott Williams \& Wilkins, Philadelphia 2001, 216-231.

37. Sporer K A: The serotonin syndrome. Implicated drugs, pathophysiology and management. Drug Saf 1995, 13:94-104. | Article I PubMed

38. Iribarne $C$, Picart $D$, Dreano $Y$ and Berthou $F$ : In vitro interactions between fluoxetine or fluvoxamine and methadone or buprenorphine. Fundam Clin Pharmacol 1998, 12:194-9. | Article | PubMed

39. World Health Organization . Cancer pain relief. With a guide to opioid availability (2 ed.) Geneva: 1996 WHO. ISBN 92-4-154482-1.

40. Meine T J, Roe M T, Chen A Y, Patel M R, Washam J B, Ohman E M, Peacock W F, Pollack C V, Jr., Gibler W B and Peterson E D: Association of intravenous morphine use and outcomes in acute coronary syndromes: results from the CRUSADE Quality Improvement Initiative. Am Heart J 2005, 149:1043-9. | Article | PubMed

41. Christie J, Markowsky S J and Valdes C: Acute trauma alters morphine clearance. J Trauma 1995, 39:749-52. | Article | PubMed

42. Jannetto $P J$ and Bratanow N C: Utilization of pharmacogenomics and therapeutic drug monitoring for opioid pain management. Pharmacogenomics 2009, 10:1157-67. | Article | PubMed

43. Dole VP and Kreek MJ. Methadone plasma levels: Sustained by a reservoir of drug in tissue. Proc Nat Acad Sci 1973, 7010. | Article

44. Hanley M J, Abernethy D R and Greenblatt D J: Effect of obesity on the pharmacokinetics of drugs in humans. Clin Pharmacokinet 2010, 49:7187. | Article | PubMed

45. Linares-Daly A. Opioid pseudoaddiction: A casualty of the war on drugs, racism, sexism, and opiophobia. Quinnipiac Health Law Journal 2012, 15:89-125. | Article

46. Schulz M and Schmoldt A: Therapeutic and toxic blood concentrations of more than $\mathbf{8 0 0}$ drugs and other xenobiotics. Pharmazie 2003, 58:447-74. | Article | PubMed

\section{Citation:}

McKeen M J and Quraishi S A: Clinical review of intravenous opioids in acute care. journal of Anesthesiology and Clinical Science 2013, 2:1. http://dx.doi.org/10.7243/2049-9752-2-1 